Stefano Salgarello, DDS

Matteo Salvadori, DDS

Francesco Mazzoleni, DDS

Viviana Salvalai, DDS

Jacopo Francinelli, DDS

Paolo Bertoletti, DDS

Daniele Lorenzi, DDS

Elisabetta Audino, DDS and

Maria Luisa Garo, MSc
CLINICAL RESEARCH

\section{Urgent Dental Care During Italian Lockdown: A Cross- sectional Survey}

\begin{abstract}
Introduction: On March 9, 2020, the Italian government imposed a nationwide lockdown to contain the spread of coronavirus disease 2019. Oral health services were limited to only essential services. Nonurgent treatments were postponed. This study described the management of urgent dental care in northern Italy during the lockdown, with a particular focus on the province of Brescia. Methods: A cross-sectional survey was administered to Italian dentists at the end of the lockdown (May 3, 2020). A total of 1407 dentists answered the survey. Results: A total of 1205 dentists were included. Most of the respondents were dental owners (73.6\%). About $79.7 \%$ of the dentists handled urgencies during the lockdown. The number of urgencies treated weekly was lower than that generally handled before the coronavirus disease 2019 outbreak $(P<.001)$. Many dentists $(81.2 \%)$ provided telephone consultations to evaluate symptoms. Pulpitis and abscesses were the most common urgencies (44.7\% and $40.2 \%$, respectively). Furthermore, $95 \%$ of dentists wore masks, protective eyewear, and surgical gloves while delivering nonpostponable treatments. The filtering facepiece 2 mask was used by $41.8 \%$ and $41.7 \%$ of owners and dental assistants, respectively. Financial concerns, risk of contagion, and personal protective equipment supply were indicated as the main current concerns. Conclusions: Our survey showed that Italian dentists took protective measures during the lockdown, thus minimizing the risk of contagion for dental health care personnel and patients. Many precautionary guidelines are now available, but a high uncertainty persists about dental health delivery during the pandemic. (J Endod 2021;47:204-214.)
\end{abstract}

\section{KEY WORDS}

Coronavirus disease 2019 lockdown; cross-sectional survey; endodontic urgencies; personal protective equipment; post-coronavirus disease 2019 concerns

On January 30,2020 , the World Health Organization (WHO) declared a global health emergency because of the spread of the severe acute respiratory syndrome coronavirus 2 (SARS-CoV-2). On February 21, 2020, the first Italian positive case was reported in the city of Codogno (Lodi, Italy). In the subsequent days, given the continuous rise of positive cases in northern Italy, the Italian government imposed a prolonged lockdown ${ }^{1}$ from March 9, 2020, to May 3, 2020.

To contain the spread of the virus and safeguard citizens, health professionals, and the whole health system, stringent safety measures were adopted ${ }^{2}$. Dental health professionals experienced an unprecedented interruption of routine dental care because of the high risk of contagion due to close contact with the patient's oropharynx and the aerosol-generating procedures ${ }^{3-5}$.

In Italy, about $93 \%$ of dental treatments, urgencies included, are handled by private dental practitioners ${ }^{6}$. Although personal protective equipment (PPE) and environment sanitization have been indicated as necessary procedures to assure high safety standards ${ }^{7}$, many concerns remain. The access to adequate PPE supplies and the time needed for cleaning and disinfection procedures might exponentially increase operating costs, thus inhibiting a safe and durable reopening of private dental offices.

During the lockdown, all dental activities were reduced to urgencies and emergencies ${ }^{8,9}$ as recommended by the American Dental Association (ADA) and principal Italian dental associations 
(National Association Italian Dentists and Italian Association Dentists). To reduce the number of treated urgencies and the use of aerosolgenerating therapies during the lockdown, the National Federation for the Orders of Doctors and Dentists further widened the ADA indications. Deferrable urgencies or confirmed or suspected coronavirus disease 2019 (COVID-19) patients were handled only by telephone or video consultation.

Pharmacologic therapy was administered only when necessary and applicable ${ }^{10}$. Instead, nonpostponable urgencies were treated using rigorous measures for preventing virus transmission ${ }^{11}$.

In March, Lombardy was the Italian area with the highest number of infected patients and deaths (approximately 89,205 infections and 16,143 deaths on a population of approximately 10,000,000 inhabitants) ${ }^{12}$. A very high rate of cases and deaths was reported in Brescia, the third Italian province hardest hit by COVID-19 $9^{13}$.

The management of urgencies by private dental practitioners during lockdown may provide essential indications for identifying effective procedures to contain the virus ${ }^{7}$ and facilitating dental activity resumption with the same standards of safety and quality as prevalent before the outbreak. The aim of this work was to understand the number of urgencies treated during the lockdown and to identify the procedures adopted by Italian private dental practitioners to minimize the risk for dental health care personnel and patients. The type of urgencies, the percentage of urgencies treated only with pharmacologic prescriptions using telehealth, and the use of PPE were investigated as necessary elements to determine future safety protocols and guidelines. An in-depth study was conducted for the province of Brescia, given the particular critical issues of COVID-19 spread in that area.

\section{MATERIALS AND METHODS}

Our study population comprised Italian dentists who worked as owners or dental associates in Lombardy dental offices. A cross-sectional survey was developed to investigate the management of urgent dental care during the Italian lockdown (March 9-May 3,2020 ). An online self-administered survey was conducted in May 2020 (Supplemental Figure $\mathrm{S} 1$ is available online at www.jendodon. com). Questionnaire responses received after May 26, 2020, were excluded from the study.

The questionnaire was administered through the SurveyMonkey (San Mateo, CA) website (https://it.surveymonkey.com). The sample of dentists was selected through the authors' mailing list and Dentists Register Commission (a specific branch of National Federation for the Orders of Doctors and Dentists) and National Association Italian Dentists Lombardy mailing lists. Participation in the questionnaire survey was voluntary, anonymous, and without any form of remuneration. Written consent was obtained from all respondents. Given the nature of this survey, no ethics committee approval was required under Italian law.

In the introduction to the survey, we explained research purposes and the responsibility of the University of Brescia for data collection and management. We specified that the project and its findings would be published in scientific articles.

Survey questions were developed after reviewing pertinent literature ${ }^{14,15}$. A preliminary question classified respondents as dental owners or dental associates. The questionnaire was designed in Italian language and comprised a series of single- or multichoice questions regarding

(1) dentists' sociodemographic characteristics,

(2) the receptivity of dental offices (ie, the number of dental chairs and the number of patients treated annually or weekly),

(3) urgencies weekly handled during lockdown and before the COVID-19 outbreak (ie, the number of urgencies and safety procedures adopted), and

(4) the number of urgencies handled in patients with confirmed or suspected COVID-19. Dentists' main concerns about the future of activity resumption were investigated through a final open question.

The questionnaire's validity was pretested on a sample of 20 dentists chosen randomly in the target population. The survey length and suitability of the questions were considered, and nonambiguity of the definitions was ensured.

Data were exported in an Excel file (Microsoft Corp, Redmond, WA) and analyzed by STATA16 (Version 16.1 for Mac; Stata Corp, College Station, TX). Comparisons were conducted between Brescia and the other Lombard provinces and between dental owners and associates. Descriptive statistics were reported as the mean \pm standard deviation (SD) for quantitative data and as frequencies and percentages for qualitative data. Given the nonnormal distribution of the variables (verified by the Shapiro-Wilk test), nonparametric tests (Mann-Whitney $U$ and Wilcoxon paired samples) were used for quantitative data. Chi-square tests were performed for qualitative data. Statistical significance was set at 5\% $(P<.05)$. Two independent authors (M.G. and M.S.) analyzed free-text responses to determine the main concerns of each respondent. Possible disagreements were resolved by consensus or discussions with a third member of the review team (S.S.). The most common concerns were reported as frequencies and percentages.

\section{RESULTS}

A total of 1487 private dental practitioners participated in the survey. One thousand two hundred five (1205) respondents were included in the study. Despite efforts to prevent skipping questions, some respondents did not answer all of the questions. It is unlikely that these minor omissions affected results.

Approximately 32.7\% (394/1205) of respondents were from the province of Brescia, and 67.3\% (811/1205) were from other provinces of Lombardy. Approximately $73.6 \%(887 / 1205)$ of respondents were dental owners, whereas $26.4 \%$ (318/1205) were dental associates who collaborated in a dental office. Thirty-one percent (31.2\%, 376/1205) were female, and $68.8 \%(829 / 1205)$ were male. The respondents' mean age was 50.3 years $(\mathrm{SD}=12.7$ years), lower in dental practitioners of Brescia (48.2 \pm 13.0 years) than in dentists of other provinces $(51.4 \pm 12.4$ years) $(z=-3.923, P<.01)$.

Overall, dental practitioners experience on average was 23.0 years (SD $=11.7$ years). A statistically significant difference emerged for this variable; respondents of Brescia had less experience than other interviewees $(z=-3.580, P$ $<$.01).

Approximately $58.7 \%(521 / 887)$ of dental owners had 2 or fewer dental chairs (Brescia: 54.7\%, 151/276; other provinces: $60.6 \%, 370 / 611)$, and $36.8 \%$ (225/611) of dental owners had 3-5 dental chairs; in Brescia, this percentage increased to $40.9 \%$ (113/276).

The annual turnover registered a statistical significance $\left(\chi_{3}^{2}=15.78, P<.01\right)$ between Brescia and the other provinces. In Brescia, 56.5\% (156/276) of dental offices handled 500-2000 patients per year followed by $34.1 \%(94 / 276)$ that treated less than 500 patients per year. In the other provinces, 47.1\% (288/611) treated 500-2000 patients 
per year, and 41.9\% (256/611) handled less than 500 patients per year; $78.7 \%$ (225/286) of dental associates worked in more than 1 dental office. All respondents' demographic characteristics were reported in Table 1.

\section{Urgency Management During Lockdown}

Approximately 80\% (79.7\%, 935/1173) of respondents handled urgencies during the COVID-19 lockdown. Percentages were higher for dental owners $(87.6 \%, 777 / 887)$ than for associates $(55.2 \%, 158 / 286)\left(\chi^{2}{ }_{1}=139.9, P<\right.$ $.001)$. The same trend and significance level emerged in Brescia and the other provinces of Lombardy. Specifically, in the province of Brescia, 90.9\% (251/276) of dental owners and $61.2 \%(63 / 103)$ of associates handled urgent dental problems $\left(\chi^{2}{ }_{1}=46.8, P<.001\right)$. In the other Lombard provinces, $86.1 \%$ (526/611) of owners handled urgencies during the lockdown, a percentage significantly higher than that handled by dental associates $\left(\chi^{2}{ }_{1}=96.5\right.$, $P<$.001). All percentages are reported in Figure 1.

The number of urgencies handled weekly during the COVID-19 lockdown was lower than that handled in any pre-COVID week (urgencies pre-coviD: $5.3 \pm 5.5$; urgencies $_{\text {in lockdown: }} 4.3 \pm 5.8 ; z=11.019$ $P<$.001). This significant reduction of urgencies during lockdown was noted both in owners and associates in Brescia and other provinces $(P<.001)$
Before COVID-19, owners had provided more urgent care per week (urgencies $_{\text {owners[pre-COVID]: }} 5.3 \pm 4.6$ ) than

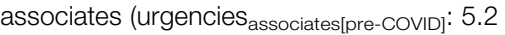
$\pm 7.6)(z=-3.169, P<.01)$. During the lockdown, no statistical significance emerged between the 2 groups, although dental owners handled a higher number of urgencies on average (urgencies owners[in lockdown] $4.5 \pm$ 6.2 , urgencies associates[in lockdown] $3.7 \pm 3.6$, $P>$.05).

In Brescia, the number of urgencies was homogenously distributed between owners and associates, both before and during the COVID-19 lockdown. In other Lombard provinces, before the COVID-19 lockdown, the number of urgencies treated weekly by dental owners was significantly higher than that treated by dental associates (urgencies owners $_{\text {s. }}$ [pre-COVID]: $5.2 \pm 4.7$, urgencies associates[preCovID]: $5.0 \pm 6.7, z=-2.764, P=.0057)$. No statistically significant difference emerged between owners and associates during the lockdown. The mean values of urgencies treated weekly were reported in Figure 2.

Fifty-three percent (381/722) of dental owners handled less than $50 \%$ of urgencies for new patients. In contrast, 32.7\% (236/722) of dental owners handled urgencies only for consolidated patients. This trend was confirmed in Brescia, where 51.7\% (121/234) of dental owners handled less than $50 \%$ of urgencies for new patients followed by $34.6 \%$ $(81 / 234)$ who treated urgencies referred only to consolidated patients. Similar percentages were registered in the other provinces.

During telephone or video consultations, $36.7 \%(265 / 722)$ of owners classified cases as urgent, representing less than $50 \%$ of patients whereas 34.6\% (250/722) identified at least $50 \%$. Twenty-one percent $(20.6 \%, 149 / 722)$ of owners considered all urgent patients. Similar percentages were registered for Brescia and other provinces. All results are summarized in Table 2.

Thirty-eight percent (37.8\%, 330/873) of dentists provided care by telephone or video consultation. Twenty percent (175/873) of respondents preferred in-office consultations. Approximately $34.4 \%$ (52/151) of associates prescribed drugs for more than $50 \%$ of cases during telephone or video consultations, whereas $18.8 \%$ (136/722) of owners prescribed drugs for more than $50 \%$ of urgencies. Similar results emerged in Brescia. Details were summarized in Figure 3.

The most frequent type of urgency was pulpitis $(44.7 \%, 390 / 873)$ followed by odontogenic and periodontal abscesses (40.2\%, 351/873). Owners and associates treated mainly pulpitis $(42.8 \%, 309 / 722$, and $53.6 \%, 81 / 151$, respectively) and odontogenic and periodontal abscesses (41.6\%, 300/722, and $33.8 \%, 51 / 151$ ) (Fig. 4). Similar percentages emerged in the province of Brescia (Fig. 4).

The clinical level of patients' oral hygiene was rated "fair" and "poor" for 61.7\% (539/

TABLE 1 - Respondents' Demographic Characteristics: Dental Office Size and Number of Collaborations for Associates

\begin{tabular}{|c|c|c|c|c|}
\hline & Lombardy $(n=1205)$ & Province of Brescia $(n=394)$ & Other provinces $(n=811)$ & $P$ value \\
\hline \multicolumn{5}{|l|}{ Sex, $n(\%)$} \\
\hline Female & $376(31.20)$ & $124(31.47)$ & $252(31.07)$ & $>.05^{\star}$ \\
\hline Male & $829(68.80)$ & $270(68.53)$ & $559(68.93)$ & \\
\hline Age, mean (SD), y & $50.3(12.7)$ & $48.2(13.0)$ & $51.4(12.4)$ & $.0001^{\dagger}$ \\
\hline Experience, mean (SD), y & $23.0(11.7)$ & $21.2(11.9)$ & $23.9(11.5)$ & $.0003^{\dagger}$ \\
\hline \multicolumn{5}{|l|}{ Dental chairs. $n(\%)^{*}$} \\
\hline 2 or less & $521(58.7)$ & $151(54.7)$ & $370(60.6)$ & $>.05^{\star}$ \\
\hline $3-5$ & $338(38.1)$ & $113(40.9)$ & $225(36.8)$ & \\
\hline $6-8$ & $19(2.1)$ & $6(2.2)$ & $13(2.1)$ & \\
\hline$\geq 9$ & $9(1.0)$ & $6(2.2)$ & $3(0.5)$ & \\
\hline \multicolumn{5}{|l|}{ Patients per year, $n(\%)^{*}$} \\
\hline$<500$ & $350(39.5)$ & $94(34.1)$ & $256(41.9)$ & $.001^{*}$ \\
\hline 500-2000 & $444(50.1)$ & $156(56.5)$ & $288(47.1)$ & \\
\hline $2000-5000$ & $85(9.6)$ & $20(7.3)$ & 65 (10.6) & \\
\hline$>5000$ & $8(0.9)$ & $6(2.2)$ & $2(0.3)$ & \\
\hline Work in 1 dental office, $n(\%)^{\ddagger}$ & $61(21.3)$ & $24(23.3)$ & $37(20.2)$ & $>.05^{*}$ \\
\hline Work in more than 1 dental office, $n(\%)^{\ddagger}$ & $225(78.7)$ & $79(76.7)$ & $146(79.8)$ & \\
\hline Treatments per day pre Covid, mean (SD) ${ }^{\ddagger}$ & $11.4(5.2)$ & $11.9(5.1)$ & $11.1(5.2)$ & $>.05^{*}$ \\
\hline
\end{tabular}

$\mathrm{SD}$, standard deviation.

Respondents: only dental owners, 887 (276 Brescia and 611 other provinces). ${ }^{*}$ Chi-square test.

${ }^{\dagger}$ Mann-Whitney $U$ test.

${ }^{\ddagger}$ Respondents: only associates, 286 (103 Brescia and 183 other provinces). 


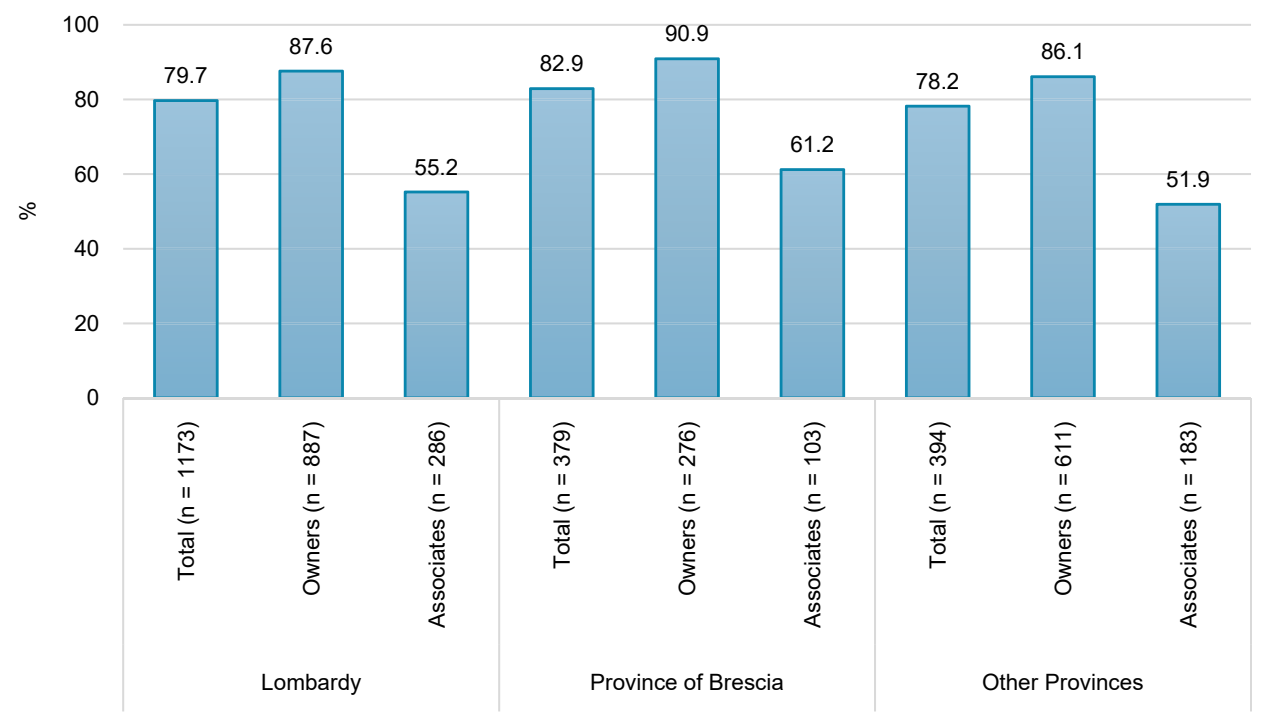

FIGURE 1 - The percentage of dentists who treated urgencies during the COVID-19 lockdown.

$873)$ and $34.8 \%(304 / 873)$ of dental practitioners, respectively. The oral hygiene was classified as "poor" mainly from associates (42.4\%, 64/151). Similar percentages emerged in Brescia (Fig. 5).

\section{PPE During Urgent Treatments}

More than $95 \%$ of dental practitioners wore surgical gloves, masks, and protective eyewear during urgent treatments; no difference emerged between owners and associates. Eighty-one percent (80.5\%, 581/
722) of owners and $88.7 \%$ (134/151) of associates wore a head covering. Disposable TNT gowns were worn by $58.6 \%$ (423/722) of owners and $52.3 \%(79 / 151)$ of associates. Overall, dental owners increased protective equipment for their dental assistants. During urgent treatments, 59.7\% (431/722) of dental assistants wore disposable TNT gowns, and 84.9\% (613/722) donned head coverings. More than $95 \%$ wore protective eyewear or a face shield, mask, and surgical gloves (Table 3).
Forty-two percent $(41.8 \%, 302 / 722)$ of owners, $27.8 \%(42 / 151)$ of associates, and $41.7 \%$ (301/722) of dental assistants wore an filtering facepiece 2 (FFP2) mask. FFP2, combined with a surgical mask, was mainly used by $41.1 \%$ (62/151) of associates (Fig. 6).

\section{Concerns About Future}

It emerged that $58.5 \%(428 / 731)$ of

respondents were worried about the following financial concerns:

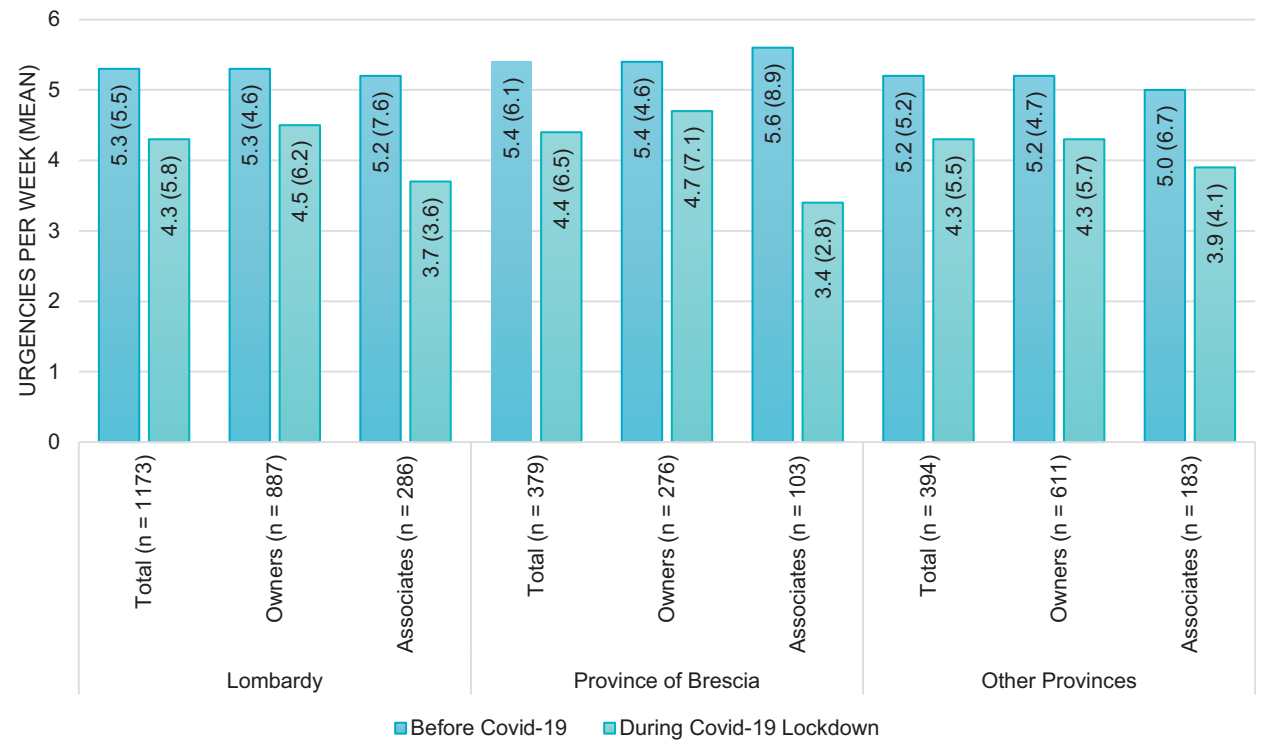

FIGURE 2 - The number of urgencies managed weekly before and during COVID-19 lockdown. Mann-Whitney Uand Wilcoxon paired samples tests were used for comparisons. Mean (SD). 
TABLE 2 - Urgency Management

\begin{tabular}{|c|c|c|c|c|}
\hline & $\begin{array}{l}\text { Total sample } \\
(N=722)\end{array}$ & $\begin{array}{l}\text { Province of Brescia } \\
\qquad(n=234)\end{array}$ & $\begin{array}{l}\text { Other provinces } \\
\quad(n=488)\end{array}$ & $P$ value \\
\hline \multicolumn{5}{|c|}{$\%$ of acute dental problems managed for new patients (\%) } \\
\hline None & $236(32.7)$ & 81 (34.6) & $155(31.8)$ & \multirow{4}{*}{$>.05^{*}$} \\
\hline Less than $50 \%$ & $381(52.8)$ & $121(51.7)$ & $260(53.3)$ & \\
\hline More than $50 \%$ & 89 (12.3) & $28(12.0)$ & $61(12.5)$ & \\
\hline All patients & $16(2.22)$ & $4(1.7)$ & $12(2.5)$ & \\
\hline \multicolumn{5}{|c|}{$\%$ of patients with urgent or emergency dental problems (\%) } \\
\hline None & $58(8.0)$ & $22(9.4)$ & $36(7.4)$ & \multirow{4}{*}{$>.05^{*}$} \\
\hline $\begin{array}{l}\text { Less than } 50 \% \text { of patients had an } \\
\text { urgency }\end{array}$ & $265(36.7)$ & $81(34 . \&)$ & $184(37.7)$ & \\
\hline $\begin{array}{l}\text { At least } 50 \% \text { of patients had an } \\
\text { urgency }\end{array}$ & $250(34.6)$ & $77(32.9)$ & $173(35.45)$ & \\
\hline All patients & $149(20.6)$ & $54(23.1)$ & 95 (19.5) & \\
\hline
\end{tabular}

Questions only for dental owners.

${ }^{*}$ Chi-square test.

(1) a decrease of profits,

(2) the rise of costs and operation time,

(3) an economic crisis due to worldwide COVID-19 spread, and

(4) patients' financial difficulties that could prevent access to dental treatments. In Brescia, 61.6\% (135/219) of the owners and 59\% (49/83) of the associates were worried about financial problems.
The risk of COVID-19 contagion was considered a concern for $28.2 \%$ (206/731) of the sample (28.2\% of owners and associates, respectively). This percentage remained unchanged for Brescia.

PPE represented a source of uncertainty for $24.8 \%$ (181/731) of dental owners. Approximately 29.8\% (71/238) of associates considered PPE to be a relevant concern.
Significant concerns were also PPE impediments, the uncertainty about PPE supply and sanitization methods, and the lack of specific guidelines to prevent contagion in dental offices. Thirteen percent (12.9\%, 94/ 731) of owners and 3.4\% (8/238) of associates showed a general sentiment of uncertainty. The primary sources of anxiety were uncertainties about bureaucratic delays because of the new adjustments in protocols,

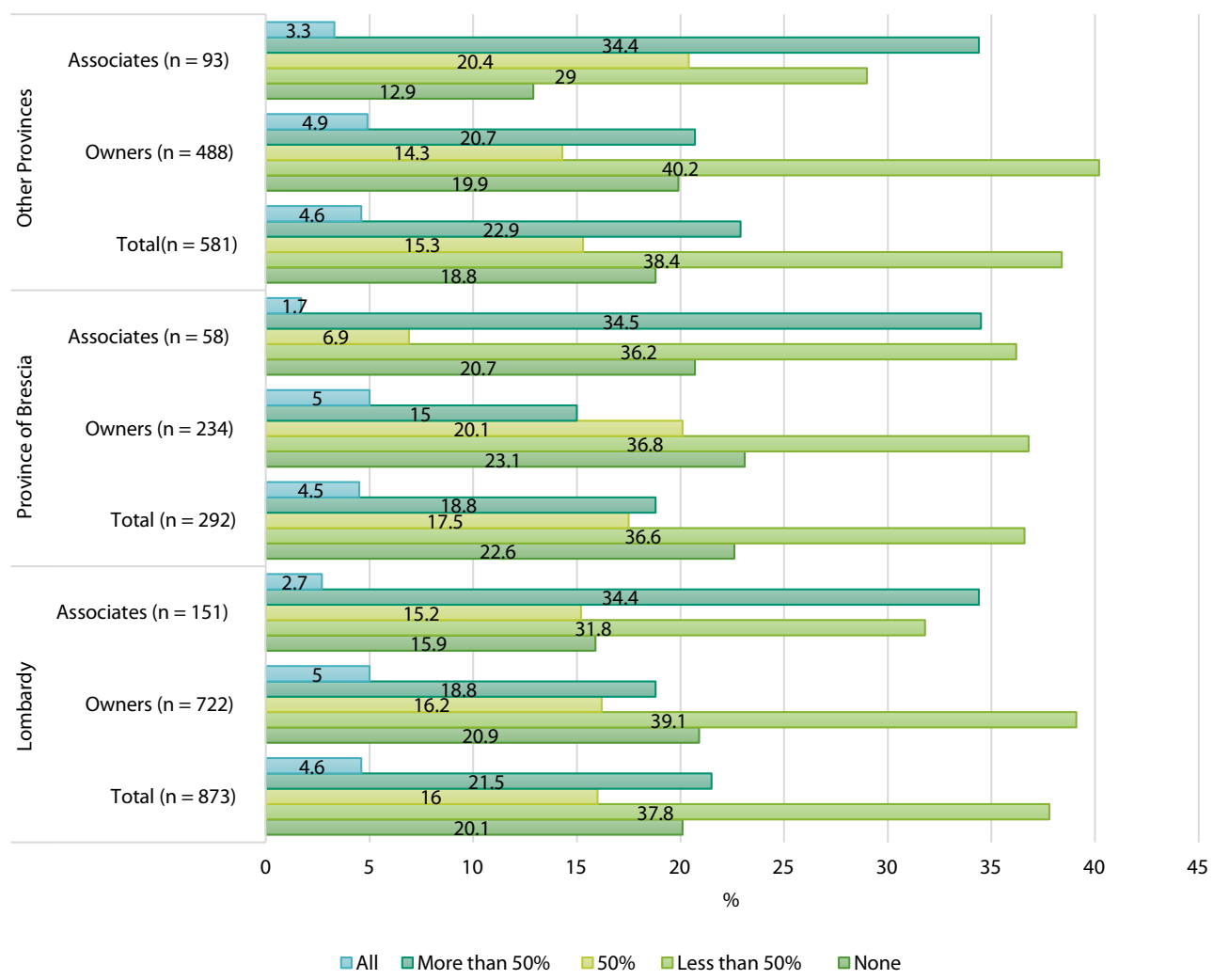

FIGURE 3 - Urgencies treated only with pharmacologic prescription (\%). 

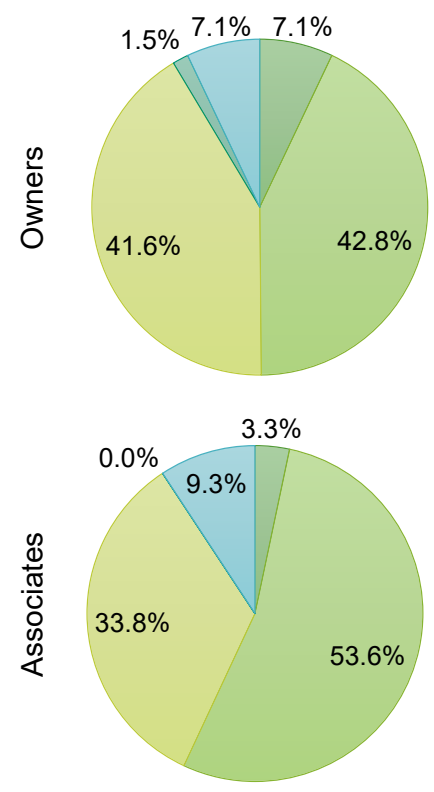

Lombardy
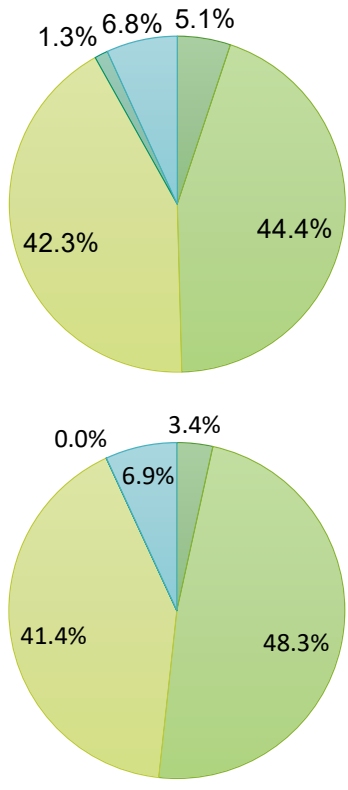

Province of Brescia
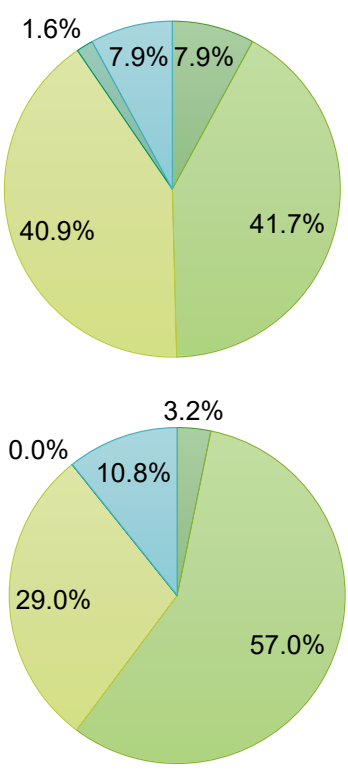

Other Provinces

FIGURE 4 - Type of urgencies (\%).

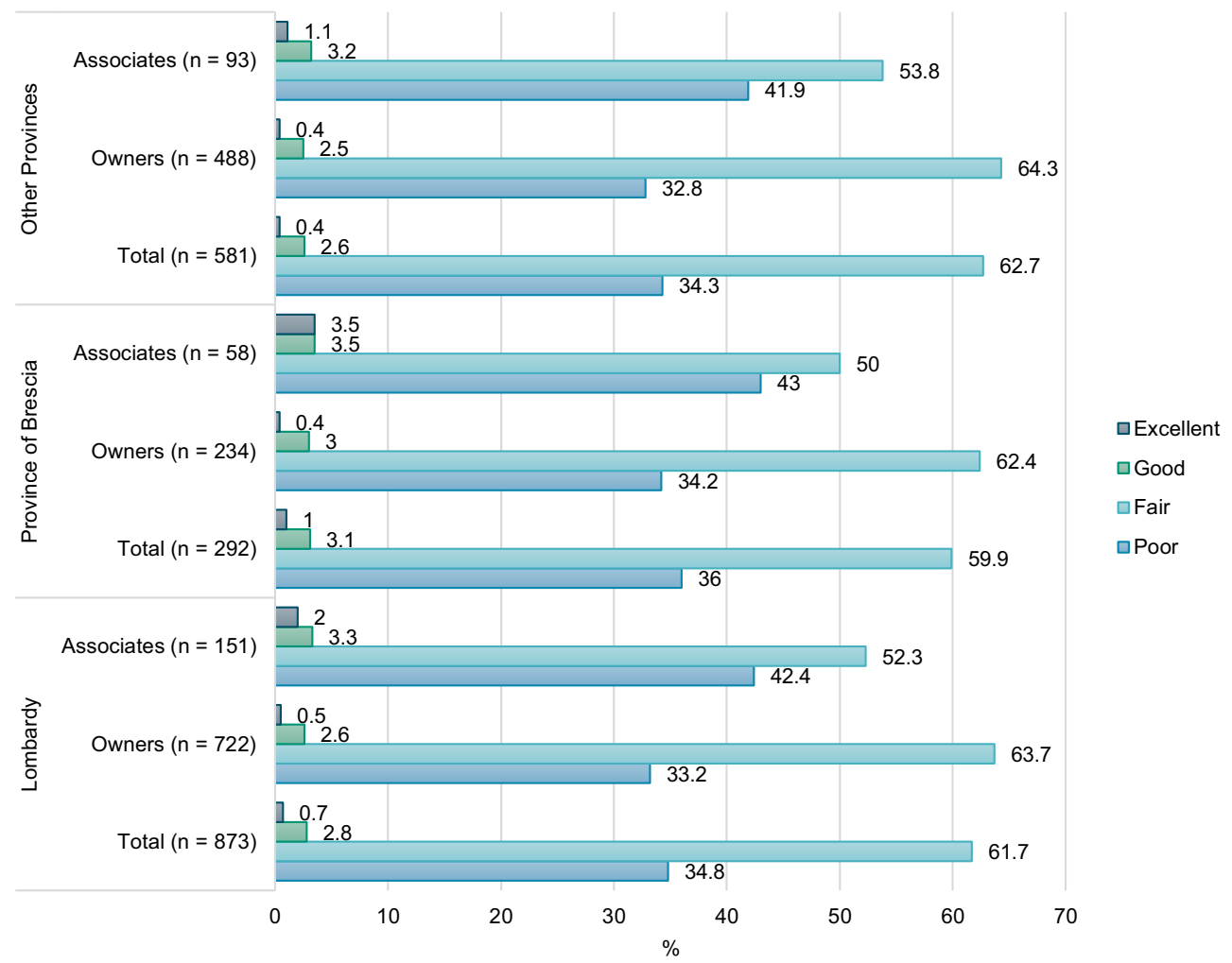

FIGURE 5 - Clinical level of oral hygiene in patients treated during lockdown (\%). 


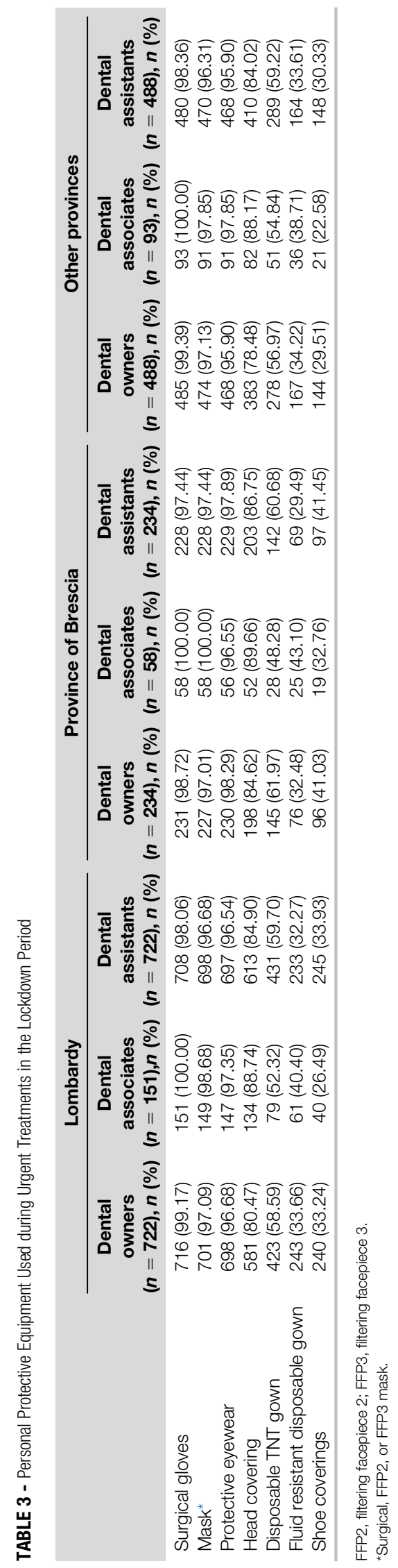

legal consequences in case of suspected contagion from patients during dental treatments, and the danger of another period of lockdown.

Ensuring high-security standards and proper dental office management was indicated by $10.7 \%$ (78/731) of owners and $8.0 \%(19 / 238)$ of associates. A small percentage of respondents $(3.3 \%, 24 / 731$, of owners and $4.6 \%, 11 / 238$, of associates) did not report any concern regarding COVID-19 contagion because of high standards of hygiene, which always characterized dental offices. All results are summarized in Figure 7 .

\section{DISCUSSION}

In Lombardy, the spread of SARS-CoV-2 virus has influenced dental activity. People have modified the use of urgency dental services, and private dental practitioners have introduced new procedures for selecting and treating patients.

This work aimed to understand the management of dental urgencies in Lombardy during the Italian lockdown to identify operative procedures and strategies for safeguarding the health of patients and dental health care personnel. A survey was conducted in May 2020. The sample was mainly composed of males (68.8\%) with a 2:1 male/female ratio, and it was explained by the higher number of male dentists in Italy ${ }^{16}$.

Overall, the number of dental urgencies significantly decreased during the lockdown compared with the pre-COVID period $(P<$ .01). In the province of Brescia, this decrease was equal to $18 \%$. Our findings have confirmed the trend already outlined in other hardest-hit areas ${ }^{17,18}$. The fear of contagion had significantly and negatively affected access to dental treatments, even in urgent cases.

The prolonged lockdown had increased pharmacologic prescription as an interim measure for postponing deferrable treatments. It could have also exposed patients to autonomous potential abuse of drugs, such as anti-inflammatory drugs and antibiotics.

Recently, the WHO warned about the issue of antibiotic overuse during the COVID-19

epidemic. Higher bacterial resistance rates and burden of disease and deaths are expected even beyond the pandemic period ${ }^{19}$. The lack of mobility ${ }^{14}$ and some elective activities (eg, periodontal and implant surgery) have reduced the need for some urgencies, such as dental trauma or bleeding.

Only $20 \%$ of dentists preferred a faceto-face consultation. More than $50 \%$ of respondents handled urgencies over telephone or video consultation, prescribing 


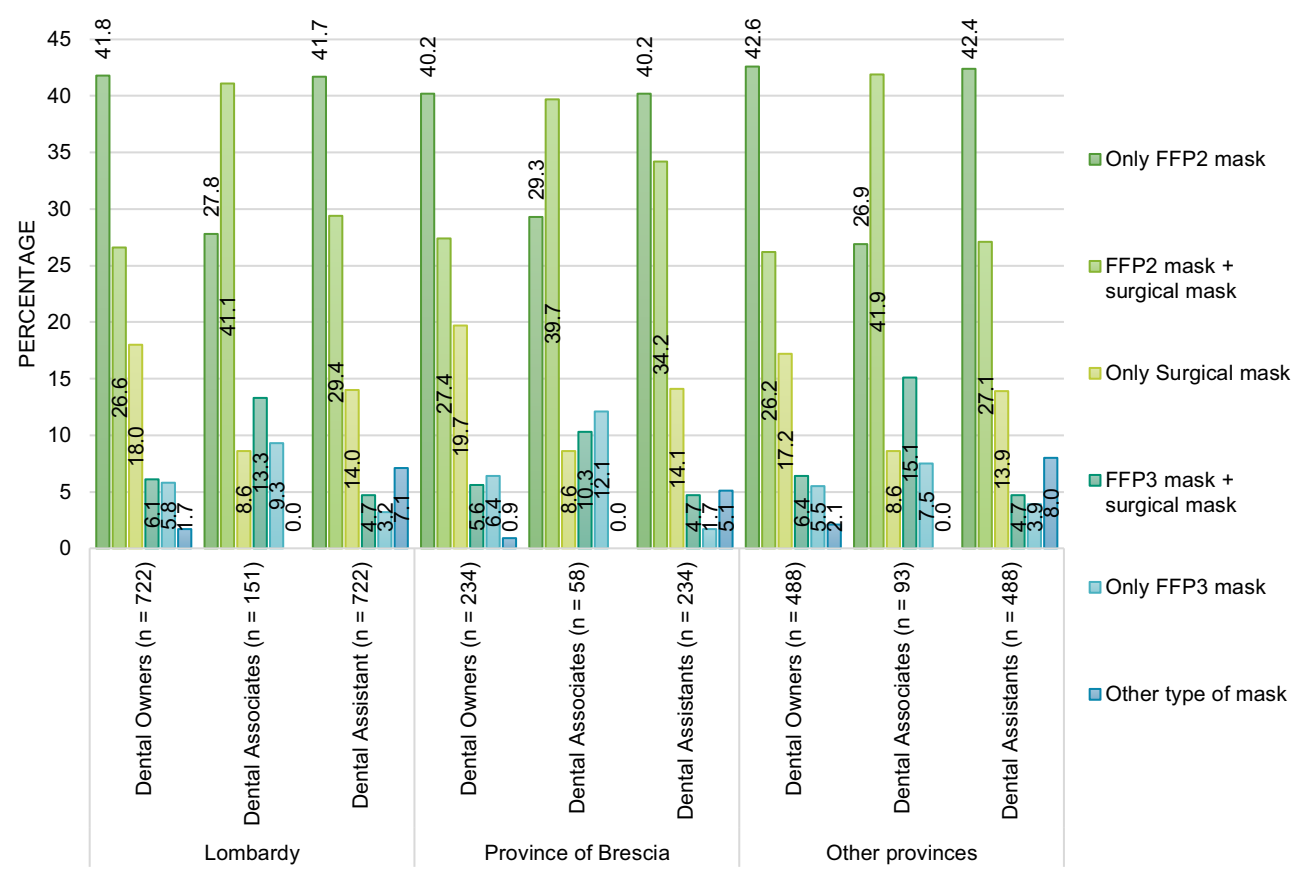

FIGURE 6 - Type of mask used during lockdown (\%).

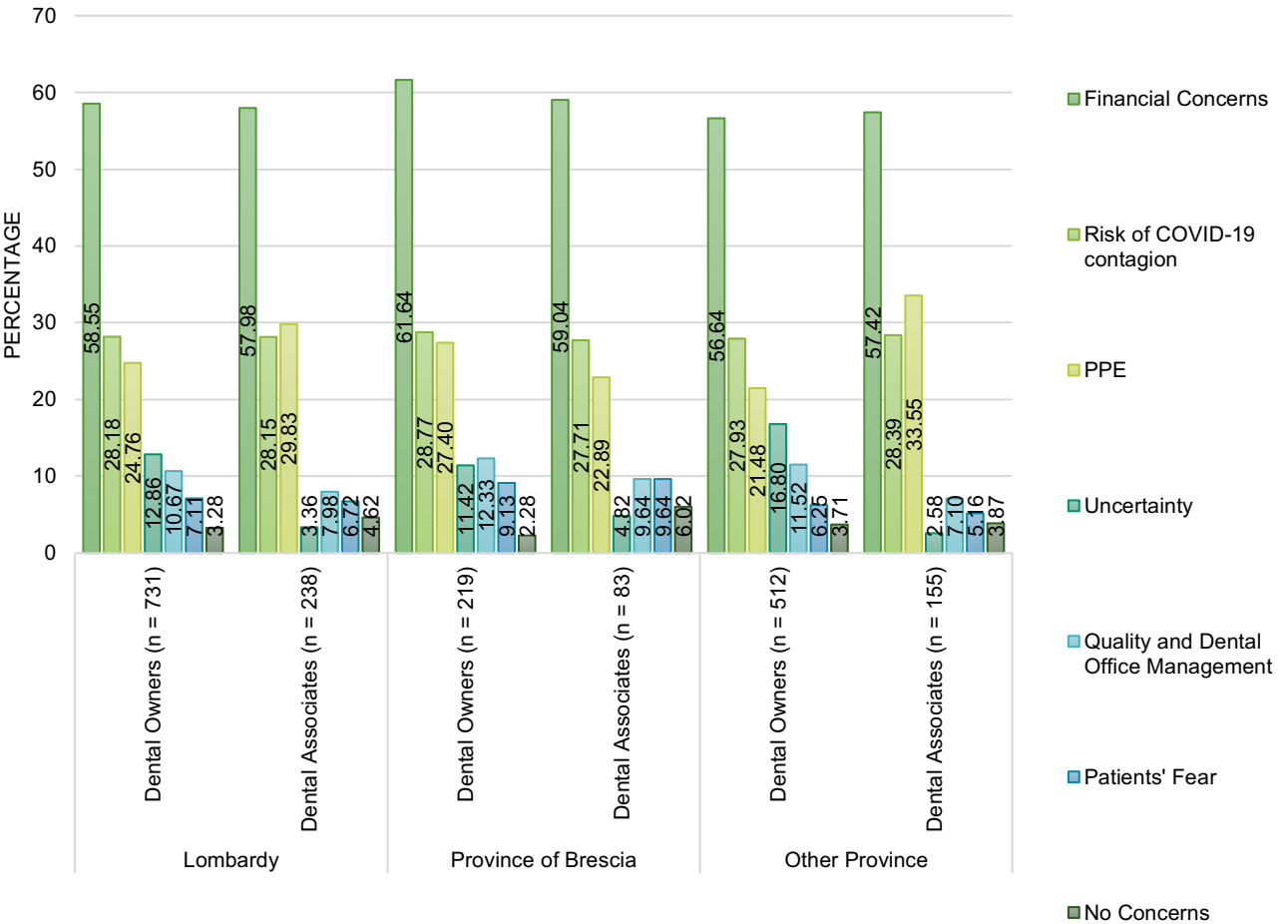

FIGURE 7 - Major concern about future (\%).

provisionally pharmacologic therapy. The significant incidence of pulpitis and abscesses already revealed in other studies $3,14,17,18,20$ could have facilitated the greater use of pharmacologic treatment.
The type of urgency and the low levels of oral hygiene registered by $34.3 \%$ of respondents have highlighted the need for a rapid resumption of all regular dental services. However, some issues might hamper a complete return to normalcy. Ninety-five percent of dentists wore the main PPE (mask, gloves, and protective glasses) during urgent treatments following the recommendations provided by the ADA, 
WHO, and Italian dental associations. Nevertheless, PPE wearing is an impediment to dental activity. Breathing difficulty, a sense of suffocation, heat stress, and the impossibility of using equipment such as a magnifying glass or surgical microscope were indicated as the main problems associated with prolonged PPE use. The lack of comfort and the increased fatigue might inhibit dentists' optimal surgical performance, as recently shown in other studies ${ }^{21}$.

To reduce the risk of infection due to aerosol production, approximately $84 \%$ of dentists (owners, 80.3\%; associates, 91.5\%; and assistants 79\%) wore an FFP2 mask alone or with a surgical mask ${ }^{22}$. Although the WHO had urged all health care professionals to rationalize protective equipment by using FFP2 masks only for contacts with confirmed or suspected COVID-19 cases $^{23}$, Italian dentists adopted more stringent measures.

The use of head coverings, protective gowns, and shoe covers was an even more debated topic. Approximately $80 \%$ of dentists wore caps, $55 \%$ wore disposable protective gowns, and only $30 \%$ wore shoe covers during urgent treatments. PPE is considered a current concern due to contradictory indications on its use, supply difficulties, and impediments to communication and visibility associated with its use.

The adoption of guidelines, risks to personnel and patients, economic and social uncertainties, increased operating costs and times, and financial concerns were considered the main concerns to restart elective procedures. Patients' financial concerns, worsened by the economic crisis, and higher costs of treatments due to increased operating time were indicated as barriers to prevent patients from accessing dental services.

Sanitization was a crucial topic. In the context of this epidemic, sanitization has been the main reason for slowing down operating times and a significant increase in treatment costs, although some dentists revealed that maintaining high standards of safety was a priority of dental offices even before the spread of SAR-CoV-2.

Aerosol production and associated contagion risks were sources of concern for $28 \%$ of dentists. This could be due to the lack of definitive and universally accepted indications regarding the actual permanence of viral load in the air after aerosol production $^{24-27}$. As recommended by the Centers for Disease Control and Prevention, routine cleaning and disinfection procedures are appropriate for infection control even in patient care areas in which aerosol-generating procedures are performed ${ }^{28}$.
The lack of a vaccine and the epidemic spread have substantial repercussions on dental activity. Thirteen percent of the dentists indicated bureaucratic or legal problems as the major concerns to restart elective procedures. The risk of new lockdowns and the lack of legislation to exclude dentists' criminal liability in the event of contagion within dental offices were often reported as crucial concerns ${ }^{29}$.

Psychological problems associated with the COVID-19 outbreak are emerging ${ }^{17}$. Global anxiety, mental health issues due to prolonged isolation (the so-called "cabin fever") $)^{30}$, and the fear of contagion during dental treatments amplify patients' anxiety ${ }^{31}$ and reduce confidence in sanitation procedures.

At present, the epidemiologic curve is increasing again in Italy. The sense of uncertainty and the consequent economic precariousness prevent a full resumption of the whole dental activity.

Current guidelines recommend ${ }^{6}$ using PPE appropriately, minimizing overlapping dental appointments, ventilating the operating rooms for at least 10-15 minutes after each appointment or consultation ${ }^{8}$, and using handpieces equipped with antireflux devices to avoid contaminations ${ }^{32}$ and encourage physical distancing

In Lombardy, dentists have rigorously adopted the WHO and Federazione Regionale degli Ordini dei Medici Chirurghi e degli Odontoiatri (Lombardy Region section) recommendations to handle dental urgencies. Besides, given the unprecedented health crisis, dentists have independently selected the most suitable operating procedures to ensure proper and safe management of urgencies. Telephone triage and consultation, in-office care only for nonpostponable urgencies, and highly safe procedures have made Italian dental offices efficient models for all areas subject to restrictions.

Dentistry is faced with several challenges. Practical and essential guidelines, better screening tests to closely monitor patients, efficient PPE supply, quick sanitization procedures, policies aimed at economic restoration, and rebalancing dental supply-demand are topics that need urgent attention.

\section{CONCLUSION}

To resume routine dental practice, dentists should improve the strengths of the dental profession by minimizing risks to patients and dental health professionals and ensuring highquality safety standards. This study allowed us to accurately describe the management of dental urgencies in the areas hardest hit.
Despite significant findings, some limitations have emerged. The questionnaire was administered during the reopening phase (called phase 2) and for a short period (only 22 days). This could have reduced the number of respondents and limited the scope of trends covered and analyzed because they are bound to have shifted with changing guidelines and measures. Selection bias and sampling error are also possible issues.

Further studies are needed to understand patients' fears and the operating procedures adopted for the new dental activity resumption phase and management of dental offices during the subsequent waves. Finally, identifying efficient, rapid, and safe operating and sanitization procedures concerning the actual stability of the virus on surfaces and in the air continues to be a priority for the dental profession as a whole during the pandemic.

\section{CREDIT AUTHORSHIP CONTRIBUTION STATEMENT}

Stefano Salgarello: Conceptualization, Methodology, Software, Resources, Validation, Writing - original draft, Supervision, Writing - review \& editing. Matteo Salvadori: Formal analysis, Data curation, Writing original draft, Project administration.

Francesco Mazzoleni: Methodology, Software, Investigation, Data curation. Viviana Salvalai: Methodology, Software, Investigation, Data curation. Jacopo Francinelli: Validation, Data curation, Writing review \& editing. Paolo Bertoletti: Conceptualization, Writing - review \& editing. Daniele Lorenzi: Methodology, Software, Investigation, Data curation. Elisabetta Audino: Supervision, Writing - review \& editing. Maria Luisa Garo: Conceptualization, Methodology, Software, Resources, Validation, Writing - original draft, Supervision, Writing - review \& editing.

\section{ACKNOWLEDGMENTS}

The authors thank all the dentists who kindly took part in this study. We are also immensely grateful to ANDI Lombardia, especially ANDI Brescia and ANDI Cremona, and CAO. We also thank all private dentists for their contribution to share our survey.

The authors deny any conflicts of interest related to this study.

\section{SUPPLEMENTARY MATERIAL}

Supplementary material associated with this article can be found in the online version at www.jendodon.com (10.1016/j.joen.2020.11. 005). 


\section{REFERENCES}

1. Governo Italiano - Presidenza del Consiglio dei Ministri. II Presidente Conte firma il Dpcm 9 marzo 2020. Available at: http://www.governo.it/it/articolo/firmato-il-dpcm-9-marzo-2020/14276. Accessed May 20, 2020.

2. Ministero degli Interni - Bilancio dei controlli Covid-19 nella fase 1: i dati del 3 maggio. Available at: https://www. interno.gov.it/it/notizie/bilancio-dei-controlli-covid-19-nella-fase-1-i-dati-3-maggio. Accessed May 20, 2020.

3. Ather A, Patel B, Ruparel NB, et al. Coronavirus disease 19 (COVID-19): implications for clinical dental care. J Endod 2020;46:584-95.

4. Peditto M, Scapellato S, Marcianò A, et al. Dentistry during the COVID-19 epidemic: an Italian workflow for the management of dental practice. Int J Environ Res Public Health 2020;17:3325.

5. Izzetti R, Nisi M, Gabriele M, et al. COVID-19 transmission in dental practice: brief review of preventive measures in Italy. J Dent Res 2020;99:1030-8.

6. Indicazioni operative per l'attività odontoiatrica durante la fase 2 della pandemia COVID-19. Available at: http://www.salute.gov.it/portale/documentazione/p6_2_2_1.jsp? lingua=italiano\&id=2917. Accessed June 1, 2020.

7. Baghizadeh Fini M. What dentists need to know about COVID-19. Oral Oncol 2020;105:104741.

8. Giudice A, Antonelli A, Bennardo F. To test or not to test? An opportunity to restart dentistry sustainably in "COVID-19 era". Int Endod J 2020;53:1020-1.

9. American Dental Association. ADA develops guidance on dental emergency, nonemergency care. Available at: https://www.ada.org/en/publications/ada-news/2020-archive/march/adadevelops-guidance-on-dental-emergency-nonemergency-care. Accessed May 18, 2020.

10. FNOMCeO: National Federation of Orders of Surgeons and Dentists. Available at: https://portale. fnomceo.it/wp-content/uploads/2020/04/COMUNICAZIONE-N-78-2020.pdf. Accessed May 20, 2020.

11. Presidenza del Consiglio dei Ministri - Decreto \#loRestoaCasa, domande frequenti sulle misure adottate dal Governo. Available at: http://www.governo.it/it/faq-iorestoacasa. Accessed May 20, 2020.

12. Lombardia notizie online. Coronavirus in Lombardia, tutti gli aggiornamenti in diretta. Available at: https://www.lombardianotizie.online/coronavirus-casi-lombardia/. Accessed June 2, 2020.

13. Brescia e Bergamo tra cultura e scienza. Available at: https://brescia.corriere.it/notizie/cronaca/ 20 maggio_17/brescia-bergamo-cultura-scienza-edf1dfa0-9843-11ea-ba09-20ae073bed63. shtml. Accessed June 16, 2020.

14. Yu J, Zhang $T$, Zhao D, et al. Characteristics of endodontic emergencies during coronavirus disease 2019 outbreak in Wuhan. J Endod 2020;46:730-5.

15. Khader Y, Al Nsour M, Al-Batayneh OB, et al. Dentists' awareness, perception, and attitude regarding COVID-19 and infection control: cross-sectional study among Jordanian dentists. JMIR Public Health Surveill 2020;6:e18798.

16. A Venezia in Salute la "valanga dei camici rosa". Available at: https://portale.fnomceo.it/a-veneziain-salute-la-valanga-dei-camici-rosa/. Accessed June 28, 2020.

17. Ahmed MA, Jouhar R, Ahmed N, et al. Fear and practice modifications among dentists to combat novel coronavirus disease (COVID-19) outbreak. Int J Environ Res Public Health 2020;17:2821.

18. Guo H, Zhou Y, Liu X, et al. The impact of the COVID-19 epidemic on the utilization of emergency dental services. J Dent Sci 2020. https://doi.org/10.1016/J.jds.2020.02.002 [Epub ahead of print].

19. WHO warns overuse of antibiotics for Covid-19 will cause more deaths. Available at: https:// www.theguardian.com/world/2020/jun/01/who-warns-overuse-of-antibiotics-for-covid-19-willcause-more-deaths. Accessed June 17, 2020.

20. Abramovitz I, Palmon A, Levy D, et al. Dental care during the coronavirus disease 2019 (COVID19) outbreak: operatory considerations and clinical aspects. Quintessence Int 2020;51:418-29.

21. Yánez Benítez C, Güemes A, Aranda J, et al. Impact of personal protective equipment on surgical performance during the COVID-19 pandemic. World J Surg 2020;44:2842-7.

22. GeZY, Yang LM, Xia JJ, et al. Possible aerosol transmission of COVID-19 and special precautions in dentistry. J Zhejiang Univ Sci B 2020;21:361-8.

23. Rational use of personal protective equipment for coronavirus disease (COVID-19) and considerations during severe shortages: interim guidance. Available at: https://apps.who.int/iris/ handle/10665/331695. Accessed June 17, 2020. 
24. Peng X, Xu X, Li Y, et al. Transmission routes of 2019-nCoV and controls in dental practice. Int J Oral Sci 2020;12:9.

25. Pyankov OV, Bodnev SA, Pyankova OG, et al. Survival of aerosolized coronavirus in the ambient air. J Aerosol Sci 2018;115:158-63.

26. van Doremalen N, Bushmaker T, Morris DH, et al. Aerosol and surface stability of SARS-CoV-2 as compared with SARS-CoV-1. N Engl J Med 2020;382:1564-7.

27. Mupparapu M. Editorial: aerosol reduction urgency in post-COVID-19 dental practice. Quintessence Int 2020;51:525-6.

28. CDC releases interim reopening guidance for dental settings. Available at: https://www.cdc.gov/ coronavirus/2019-ncov/hcp/infection-control-recommendations.html. Accessed June 30, 2020 .

29. INAIL. L'infortunio sul lavoro per Covid-19 non è collegato alla responsabilità penale e civile del datore di lavoro. Available at: https://www.inail.it/cs/internet/comunicazione/sala-stampa/comunicatistampa/com-stampa-responsabilita-datore-lavoro-infortunio-covid-19.html. Accessed May 25, 2020.

30. Tonks A. Cabin fever. BMJ 2008;336:584-6.

31. Freeman R. Barriers to accessing dental care: patient factors. Br Dent J 1999;187:141-4.

32. Spagnuolo G, De Vito D, Rengo S, et al. COVID-19 outbreak: an overview on dentistry. Int J Environ Res Public Health 2020;17:2094. 